\title{
Planar LAAPDs: temperature dependence, performance, and application in low-energy X-ray spectroscopy
}

L. Ludhovaa,b,*, F.D. Amaro ${ }^{\text {c }}$, A. Antognini ${ }^{\mathrm{d}}$, F. Biraben ${ }^{\mathrm{e}}$, J.M.R. Cardoso ${ }^{\mathrm{c}}$, C.A.N. Conde ${ }^{c}$, D.S. Covita ${ }^{c}$, A. Dax ${ }^{\text {f }}, 1$, S. Dhawan ${ }^{\text {f }}$, L.M.P. Fernandes ${ }^{\text {, }}$, T.W. Hänsch ${ }^{\mathrm{d}}$, V.-W. Hughes ${ }^{\mathrm{f}}$, O. Huot ${ }^{\mathrm{a}}$, P. Indelicato ${ }^{\mathrm{e}}$, L. Julien ${ }^{\mathrm{e}}$, P.E. Knowles ${ }^{\mathrm{a}}$, F. Kottmann ${ }^{\text {g }}$, J.A.M. Lopes ${ }^{\text {c }}$, Y.-W. Liu' ${ }^{\text {h }}$ C.M.B. Monteiro ${ }^{\text {c }}$, F. Mulhauser ${ }^{\mathrm{a}, 2}$, F. Nez ${ }^{\mathrm{e}}$, R. Pohl ${ }^{\mathrm{b}, \mathrm{d}}$, P. Rabinowitz ${ }^{\mathrm{i}}$, J.M.F. dos Santos ${ }^{\mathrm{c}}$, L.A. Schaller ${ }^{\mathrm{a}}$, D. Taqqu ${ }^{\text {b }}$ J.F.C.A. Veloso ${ }^{c}$

${ }^{a}$ Département de Physique, Université de Fribourg, CH-1700 Fribourg, Switzerland

${ }^{\mathrm{b}}$ Paul Scherrer Institut, CH-5232 Villigen PSI, Switzerland

${ }^{\mathrm{c}}$ Departamento de Física, Universidade de Coimbra, PT-3000, Coimbra, Portugal

${ }^{\mathrm{d} M a x-P l a n c k}$ Institut für Quantenoptik, D-85748 Garching, Germany

${ }^{e}$ Laboratoire Kastler Brossel, École Normale Supérieure et Université P. et M. Curie, F-75252 Paris, Cedex 05, France

${ }^{\mathrm{f}}$ Physics Department, Yale University, New Haven, CT 06520-8121, USA

${ }^{\mathrm{g}}$ Labor für Hochenergiephysik, ETH-Hönggerberg, CH-8093 Zürich, Switzerland

${ }^{\mathrm{h}}$ Physics Department, National Tsing Hua University, Hsinchu 300, Taiwan

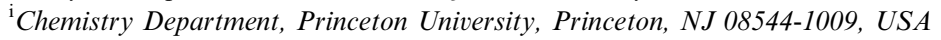

Received 1 November 2004; accepted 4 November 2004

Available online 16 December 2004

\begin{abstract}
An experiment measuring the $2 \mathrm{~S}$ Lamb shift in muonic hydrogen $\left(\mu^{-} \mathrm{p}\right)$ was performed at the Paul Scherrer Institute, Switzerland. It required small and compact detectors for $1.9 \mathrm{keV}$ X-rays (2P-1S transition) with an energy resolution around $25 \%$ at $2 \mathrm{keV}$, a time resolution better than $100 \mathrm{~ns}$, a large solid angle coverage, and insensitivity to a $5 \mathrm{~T}$ magnetic field. We chose Large Area Avalanche Photodiodes (LAAPDs) from Radiation Monitoring Devices as X-ray detectors, and they were used during the last data taking period in 2003. For X-ray spectroscopy applications, these LAAPDs have to be cooled in order to suppress the dark current noise; hence, a series of tests were performed to choose the optimal operation temperature. Specifically, the temperature dependence of gain, energy resolution, dark current,
\end{abstract}

\footnotetext{
*Corresponding author. Paul Scherrer Institut, CH-5232, Villigen PSI, Switzerland. Tel.: +41 56310 3758; fax: +4156310 5230.

E-mail address: livia.ludhova@psi.ch (L. Ludhova).

${ }^{1}$ Present address: CERN, CH-1211 Geneva 23, Switzerland.

${ }^{2}$ Present address: University of Illinois at Urbana-Champaign, IL 61801, USA.
} 
excess noise factor, and detector response linearity was studied. Finally, details of the LAAPDs application in the muonic hydrogen experiment as well as their response to $\alpha$ particles are presented.

(C) 2004 Elsevier B.V. All rights reserved.

PACS: 07.85.-m; 29.40.Wk; 85.60.Dw; 36.10.Dr

Keywords: Low-energy X-ray spectroscopy; Large Area Avalanche Photodiodes performance; Temperature dependence; Response to $\alpha$ particles

\section{Introduction}

An experiment measuring the $2 \mathrm{~S}$ Lamb shift $\Delta E(2 \mathrm{P}-2 \mathrm{~S})$ in muonic hydrogen $\left(\mu^{-} \mathrm{p}\right)$ by precision laser spectroscopy was being performed at the Paul Scherrer Institute (PSI), Switzerland [1]. The experiment required the detection of $1.9 \mathrm{keV} \mathrm{X-}$ rays from the muonic hydrogen $\mathrm{K}_{\alpha}$ Lyman line. The apparatus was installed in a muon beam area at the PSI proton accelerator, an environment with a rather high level of neutron-induced radiative processes as well as electromagnetic and acoustic noise.

The $1.9 \mathrm{keV} \mathrm{X}$-ray detector has to reach an energy resolution of $\sim 25 \%$ and a time resolution better than $100 \mathrm{~ns}$. To optimize the solid angle for $\mathrm{X}$-rays, the detector has to be mounted as near as possible to the pencil-shaped volume where the $\mu^{-} p$ atoms are formed. There is space for two sets of X-ray detectors (with sensitive areas up to $\sim 2 \times 17 \mathrm{~cm}^{2}$ ) at the top and bottom side of the gas target, which is mounted inside a solenoid with $20 \mathrm{~cm}$ inner diameter. The magnetic field of $5 \mathrm{~T}$ produced by the solenoid is another limitation for the detector choice. In addition, the whole target and detector setup is operated in vacuum.

The experiment was installed for the first time in the muon beam area during an engineering run in 2002. A second beam period followed in 2003 during which the apparatus was further improved. Valuable data were taken in the last few weeks of the 2003 run, with the aim to search for the $2 \mathrm{P}-2 \mathrm{~S}$ resonance.

In 2002 we used Large Area Avalanche Photodiodes from Advanced Photonix Inc. [2] (API LAAPDs), a representative of the beveled-edge LAAPDs, reviewed, for example, in Ref. [3]. They are circular, with a $16 \mathrm{~mm}$ diameter active surface surrounded by an $\sim 5 \mathrm{~mm}$ wide ring of inactive material (Fig. 1). Their behavior in high magnetic fields was studied in Refs. [4,5], while a systematic investigation of their low-temperature performance in X-ray and visible-light detection can be found in Ref. [6]. An example of their application to muonic atom spectroscopy during the first stages of our experiment is given in Ref. [7].

During the most recent measurements in 2003, we replaced the API diodes by LAAPDs from Radiation Monitoring Devices Inc. [8] (RMD LAAPDs). Two arrays of these detectors mounted below and above the pencil-shaped target have a considerably larger solid angle for X-ray detection due to the LAAPD square shape and better ratio of sensitive to total detector area (Fig. 1). The principle of the RMD LAAPDs, in particular their novel planar bevel fabrication process, is summarized in Ref. [9]. In Section 2 we describe these LAAPDs, their performance and the results of

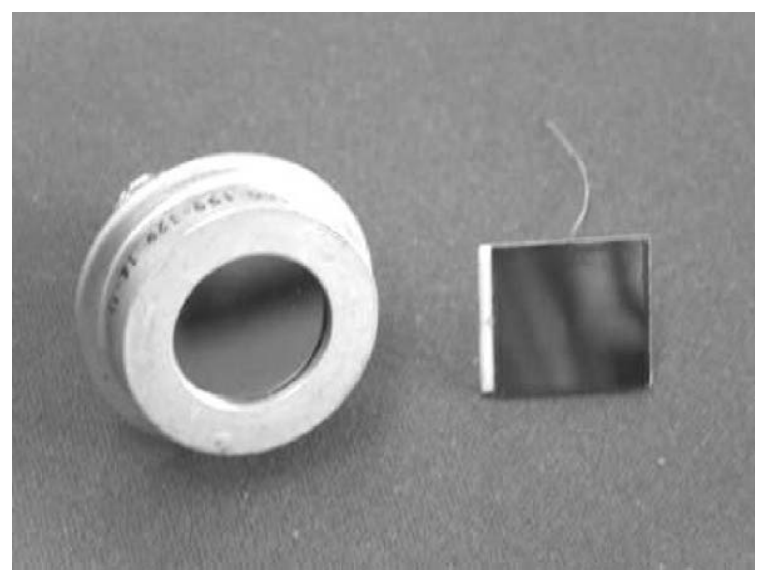

Fig. 1. Circular beveled-edge API LAAPD (left) with $16 \mathrm{~mm}$ diameter active surface area and square planar RMD LAAPD (right) with $13.5 \times 13.5 \mathrm{~mm}^{2}$ active surface. 
systematic tests. Section 3 contains some details about the RMD LAAPDs application in our experiment, in particular their response to $\alpha$ particles. A comparison with the previously used API LAAPDs is given in the conclusions.

\section{Properties of the RMD LAAPDs}

\subsection{First tests}

RMD LAAPDs, model S1315, are square planar devices with a $13.5 \mathrm{~mm} \times 13.5 \mathrm{~mm}$ sensitive area surrounded by $1 \mathrm{~mm}$ wide borders of inactive material. The operational voltage indicated by the manufacturer is in the region from 1560 to $1765 \mathrm{~V}$ at $23^{\circ} \mathrm{C}$. The first tests with a ${ }^{55} \mathrm{Fe}$ source emitting $5.9 \mathrm{keV} \mathrm{X}$-rays performed at temperatures above $0^{\circ} \mathrm{C}$ have shown that these LAAPDs require cooling to temperatures well below $0^{\circ} \mathrm{C}$ in order to detect low-energy $\mathrm{X}$-rays with an acceptable resolution. The main reason is a high dark current reaching $1 \mu \mathrm{A}$ at $1620 \mathrm{~V}$ at room temperature; when cooled to $1^{\circ} \mathrm{C}, 1 \mu \mathrm{A}$ is reached at $1710 \mathrm{~V}$ (Fig. 2).

At room temperature, the noise tail in the energy spectrum extends up to $\sim 6 \mathrm{keV}$. By cooling below $-20^{\circ} \mathrm{C}$, satisfactory results were obtained not only for $5.9 \mathrm{keV}$ X-rays, but also for $2.3 \mathrm{keV}$ $\mathrm{X}$-rays, for which an energy resolution of $26 \%$ FWHM was reached. The $2.3 \mathrm{keV} \mathrm{X}$-rays were produced by sulfur fluorescing when irradiated by

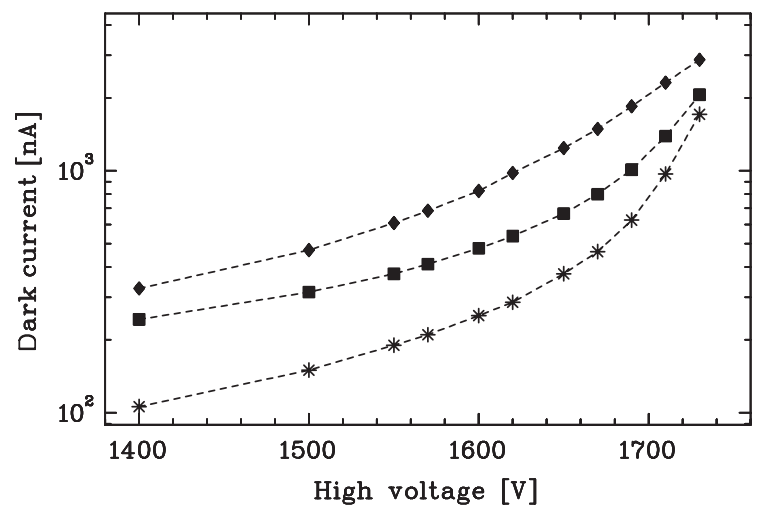

Fig. 2. LAAPD dark current versus high voltage measured at $1^{\circ} \mathrm{C}(*), 10^{\circ} \mathrm{C}(\boldsymbol{\square})$, and $23^{\circ} \mathrm{C}(\bullet)$.

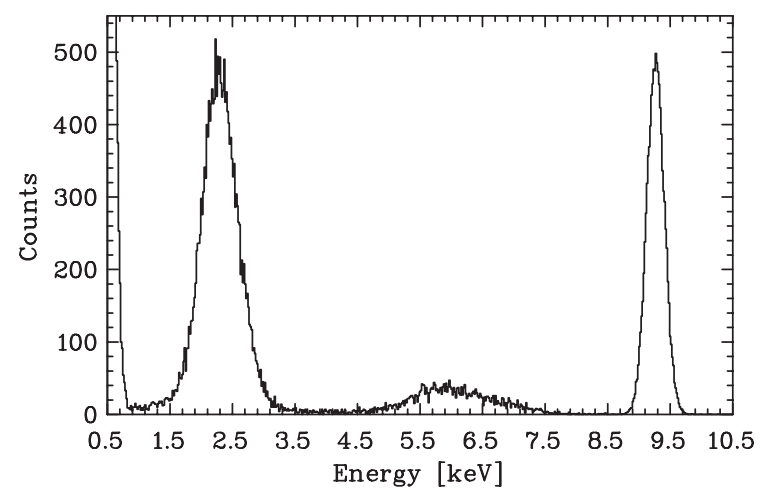

Fig. 3. LAAPD energy spectrum measured at $-23^{\circ} \mathrm{C}$ with 2.3 and $5.9 \mathrm{keV} \mathrm{X}$-ray peaks and a peak due to the test pulses from a pulse generator.

a strong ${ }^{55} \mathrm{Fe}$ source. An example of such a spectrum measured at $-23^{\circ} \mathrm{C}$ is shown in Fig. 3, where 2.3 and $5.9 \mathrm{keV} \mathrm{X}$-ray peaks, as well as a peak due to the test pulses from a pulse generator, which are fed directly to the preamplifier, are visible. The noise tail ends at $0.9 \mathrm{keV}$, which makes $\mathrm{X}$-ray spectroscopy around $2 \mathrm{keV}$ well feasible.

\subsection{Experimental setup}

In order to choose the optimal working temperature and to better understand the temperature dependence of the RMD LAAPD performance both for X-ray and visible-light detection, a series of tests were performed. X-rays with energies up to $15 \mathrm{keV}$ from ${ }^{54} \mathrm{Mn},{ }^{55} \mathrm{Fe}$, and ${ }^{57} \mathrm{Co}$ radioactive sources were used. Light pulses from a light emitting diode (LED) with a peak emission at $635 \mathrm{~nm}$ were carried by a light guide to the LAAPD surface. The LED light intensity was varied to achieve an energy equivalent (LAAPD pulse height) of $11-14.5 \mathrm{keV}$.

The LAAPDs were operated in a light-tight, thermally insulated box. A constant flow of cold nitrogen gas, regulated by a heater submerged in a container of liquid nitrogen, was used for LAAPD cooling with a temperature stabilization within $\pm 0.5^{\circ} \mathrm{C}$.

The LAAPD signals were fed into a low-noise charge-sensitive integrating RAL 108A preamplifier [10] followed by a linear amplifier HP 5582A, for which a gain between 16 and 128 as well as a 
$200 \mathrm{~ns}$ shaping time constant were used. A PCbased, 1024-channel analyzer Nucleus PCA II was used to record the energy spectra.

\subsection{Gain measurements}

Absolute gain measurements rely on the determination of unity gain, which was found by averaging the amplitudes of $100 \mathrm{~ns}$ visible-light LED pulses (635 nm wavelength) measured at a bias voltage in the range from 300 to $500 \mathrm{~V}$. For bias voltages below $300 \mathrm{~V}$ the recombination of the primary electron-hole pairs plays an important role and the absolute gain is below one. Fig. 4 shows the relative amplitudes of the light pulses as a function of high voltage, together with the dark current observed during the measurement. A horizontal line shows the unity gain.

Assuming that the visible-light and the X-ray gains are equal at low LAAPD gains, the absolute gain for X-rays was determined with a ${ }^{55} \mathrm{Fe}$ source emitting $5.9 \mathrm{keV} \mathrm{X}$-rays. Absolute $\mathrm{X}$-ray gain versus high voltage, for the temperature range from $-46^{\circ} \mathrm{C}$ to $17^{\circ} \mathrm{C}$, is given in Fig. 5 .

For a given bias voltage, the gain increases exponentially with decreasing temperature as seen in Fig. 6. The dependency is more pronounced for higher bias voltages and similar for both X-rays (solid lines) and visible light (dashed lines). Below a certain temperature the gain starts to increase even more rapidly, as is demonstrated in Fig. 6.

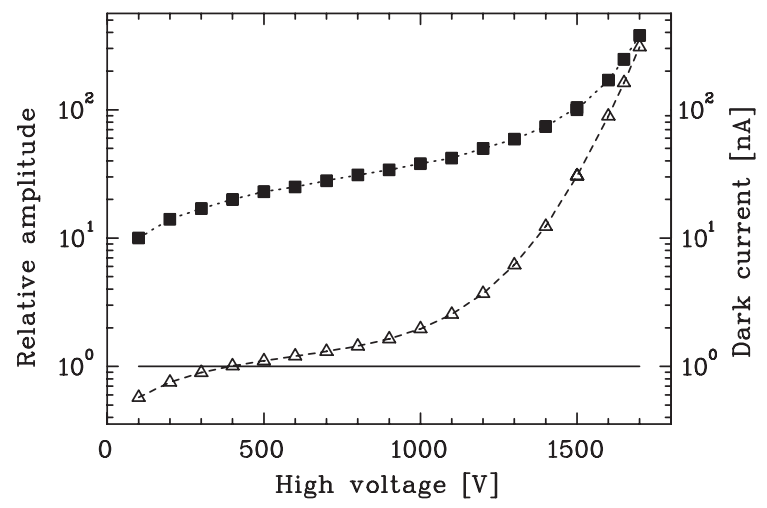

Fig. 4. LAAPD relative amplitude of the visible-light pulses $(\triangle)$ and the dark current $(\boldsymbol{\square})$ versus high voltage; measurement performed at $7^{\circ} \mathrm{C}$. The horizontal line represents the unity gain.

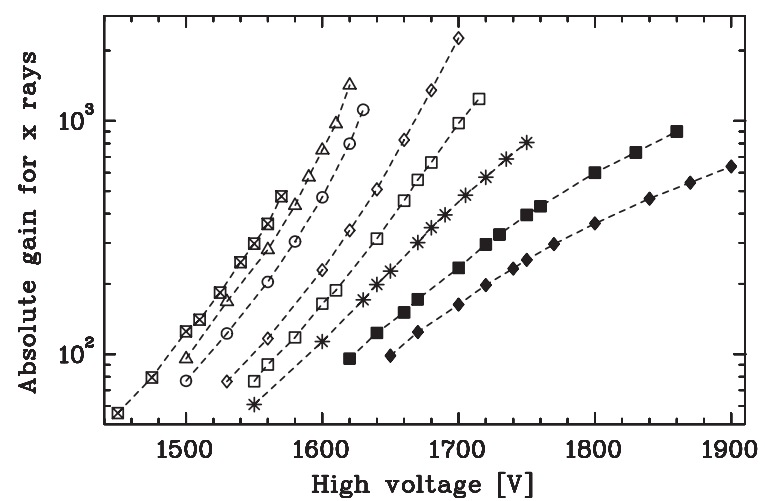

Fig. 5. LAAPD absolute $\mathrm{X}$-ray gain versus high voltage measured at $-46(\otimes),-40(\triangle),-33(\bigcirc),-17(\diamond),-8(\square)$, $0(*), 10(\mathbf{\square})$, and $17^{\circ} \mathrm{C}(\bullet)$.

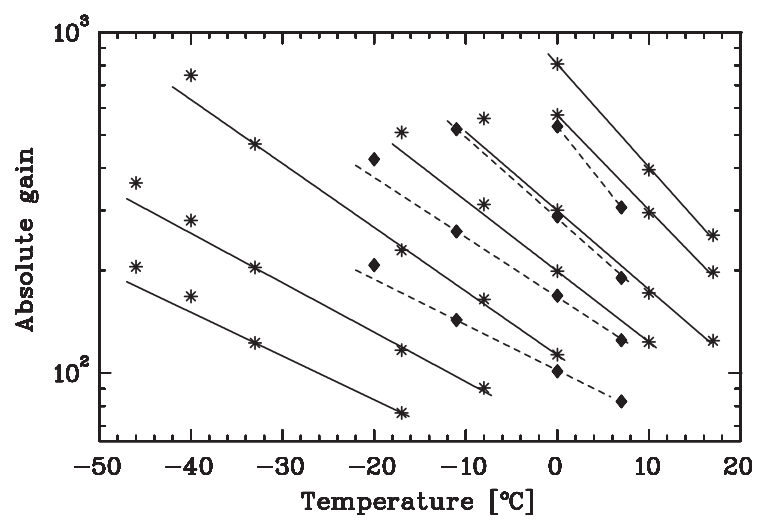

Fig. 6. LAAPD absolute X-ray $(*)$ and visible-light $(\diamond)$ gain versus temperature. Measurements were performed at constant high voltages with $\mathrm{X}$-rays (solid lines) at (from right to left) $1530,1560,1600,1640,1670,1720$, and $1750 \mathrm{~V}$ and with visible light (dashed lines) at 1590, 1625, 1660, and $1700 \mathrm{~V}$.

\subsection{Energy resolution}

At low gain values the energy resolution improves with increasing gain. This trend continues up to a gain around 200 where the optimum is obtained, for both $5.9 \mathrm{keV} \mathrm{X-rays} \mathrm{(Fig.} \mathrm{7)} \mathrm{and}$ visible light (Fig. 8). This optimal gain value does not depend on the temperature. Higher gain increases the effect of spatial nonuniformity of the LAAPD gain. Due to the local character of the $\mathrm{X}$-ray interaction with an LAAPD, this effect worsens the X-ray energy resolution. For light 


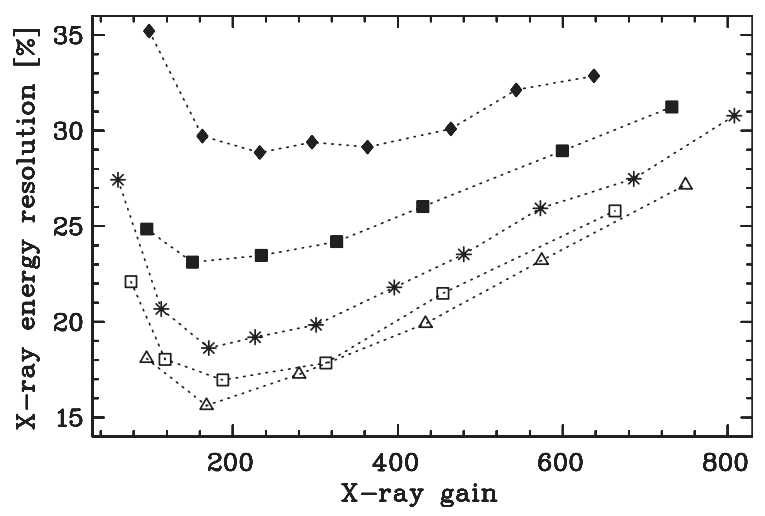

Fig. 7. LAAPD energy resolution for $5.9 \mathrm{keV} \mathrm{X}$-rays versus gain measured at $-40(\triangle),-8(\square), 0(*), 10(\mathbf{\square})$, and $17^{\circ} \mathrm{C}(\diamond)$.

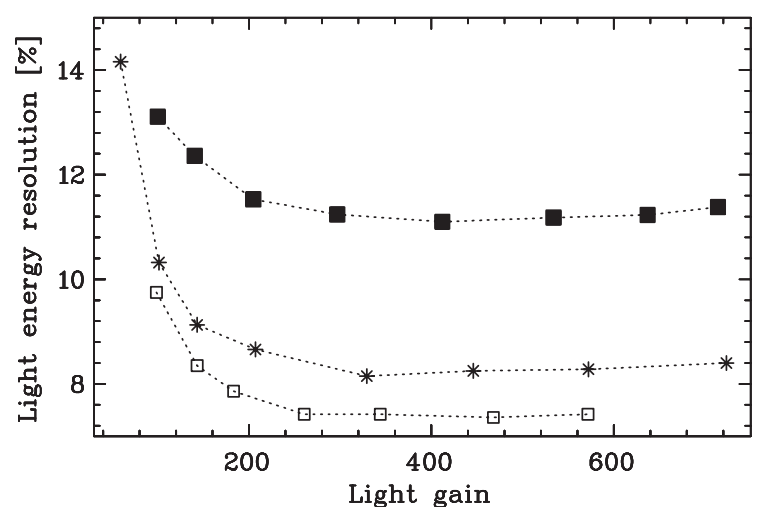

Fig. 8. LAAPD energy resolution for visible light versus gain measured at $-12(\square), 0(*)$ and $10^{\circ} \mathrm{C}(\mathbf{\square})$. The energy equivalent of the light pulses corresponds to $11-14.5 \mathrm{keV}$ X-rays.

detection the whole illuminated area contributes to the output signal, averaging local gain variations [11]. Consequently and in contrast to the X-rays, the visible-light energy resolution remains constant at gains above 250 .

Simultaneous measurements of $5.4 \mathrm{keV}$ X-rays, visible-light pulses, and test signals from a pulse generator were made at different gains and temperatures. The energy calibration was determined by the X-ray peak and the LAAPD gain was deduced from the position of the visible-light peak. The position of the test-pulse peak does not depend on the LAAPD gain. Its width represents

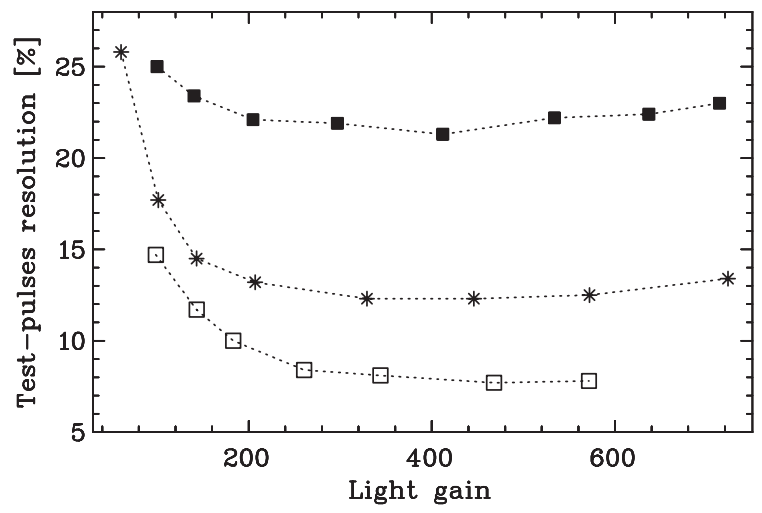

Fig. 9. LAAPD energy resolution for the test pulses versus gain, normalized to $5.9 \mathrm{keV}$. Measurements at $-12(\square), 0(*)$, and $10^{\circ} \mathrm{C}(\mathbf{\square})$.

the LAAPD dark current and preamplifier noise contributions to the overall resolution [3]. To express the test-pulse width as a relative energy resolution, it has to be normalized to a given energy which was chosen to be $5.9 \mathrm{keV}$, as shown in Fig. 9. Also here, the resolution reaches the minimum at a gain of about 200 and stabilizes for higher gains. The similar behavior of the visiblelight and test-pulse resolutions is explained in Section 2.6.

\subsection{Dark current}

The dark current depends strongly on the temperature and LAAPD gain. At a given temperature it increases with gain and is reduced by an order of magnitude for each $20^{\circ} \mathrm{C}$ temperature decrease, as shown in Fig. 10. At $-33^{\circ} \mathrm{C}$ and in the gain region below 800 , the dark current is below $10 \mathrm{nA}$.

\subsection{Excess noise factor}

Measurements of the $5.4 \mathrm{keV}$ X-rays from a ${ }^{54} \mathrm{Mn}$ source, visible-light, and test pulses were made simultaneously for different temperatures and different gains, allowing us to study the temperature dependence of the excess noise factor $(F)$, defined [3] as

$F=1+\sigma_{A}^{2} / M^{2}$, 


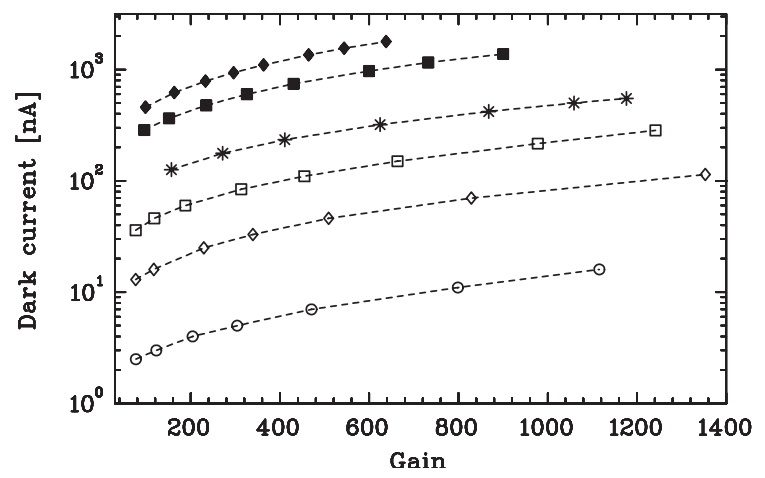

Fig. 10. LAAPD dark current versus gain measured at -33 $(\bigcirc),-17(\diamond),-8(\square), 0(*), 10(\mathbf{\square})$, and $17^{\circ} \mathrm{C}(\diamond)$.

where $M$ is the LAAPD gain and $\sigma_{A}$ its variance. Neglecting the small light-intensity fluctuations and considering that detector inhomogeneity contributions to the energy resolution are averaged out for the light measurements, the energy resolution $\Delta E(\mathrm{FWHM})$ of the light peak with a Gaussian shape can be described [3] as

$\Delta E^{2}=(2.355)^{2} F E \varepsilon+\Delta_{\text {noise }}^{2}$,

where $E$ is the energy equivalent of the light peak, $\varepsilon$ is the energy per electron-hole pair creation in $\mathrm{Si}$ $(3.6 \mathrm{eV})$, and $\Delta_{\text {noise }}^{2}$ is the dark noise contribution of the diode-preamplifier system.

In the energy spectrum, the X-ray peak gives the energy calibration. The FWHM of the test pulses peak defines $\Delta_{\text {noise }}^{2}$. The deduced value of $F$ was found to be temperature independent and slowly increasing with the LAAPD gain (Fig. 11). A faster increase at gains above 300 reflects the contribution of holes to the amplification process [11]. Typical values were $F \approx 2.2$ at gain 200 and $F \approx 2.8$ at gain 600 ; this represents a $27 \%$ increase.

As given by Eq. (2), for light pulses with an energy equivalent $E$ there are two contributions to the resolution $\Delta E$. By cooling, the contribution from the dark current noise $\Delta_{\text {noise }}^{2}$ is suppressed (Fig. 9) and the contribution due to the temperature independent increase of the excess noise factor becomes relatively more significant. However, it is important to note that a $27 \%$ increase of $F$ is accompanied by only a small increase of $\Delta E$ even at low temperatures. For light pulses with an

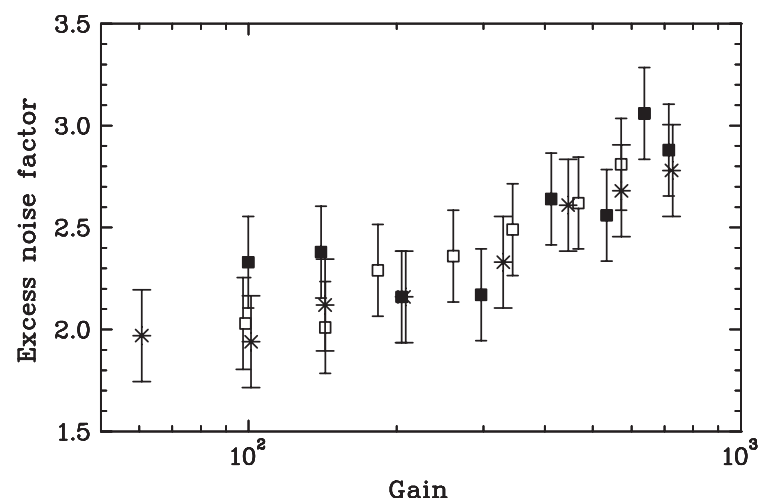

Fig. 11. LAAPD excess noise factor versus gain measured at $-12(\square), 0(*)$, and $10^{\circ} \mathrm{C}(\mathbf{\square})$.

energy equivalent in the range $11-14.5 \mathrm{keV}$, the corresponding $\Delta E$ increase is below $4 \%, 7 \%$, and $10 \%$ at temperatures 10,0 , and $-12^{\circ} \mathrm{C}$, respectively. This also explains the similar behavior of the visible-light and test-pulse resolutions as functions of LAAPD gain, as was shown in Section 2.4 and in Figs. 8 and 9.

\subsection{Nonlinearity}

The use of the X-ray peak for the energy calibration of the light peak is correct only if the LAAPD response is perfectly linear, i.e., if the resulting signal amplitude is proportional to the initial number of electron-hole pairs. In reality, there is a well-known nonproportionality between the gains for X-ray and visible-light events, as well as between X-ray events with different energies [3,6,11-13]. In contrast to visible light, an X-ray interacting in the LAAPD produces high charge densities causing both a decrease of the local electric field and local heating. The effect is important at higher gains and causes the X-ray gain to be smaller than the visible-light gain.

The nonlinearity for X-rays with different energies was measured with a ${ }^{57} \mathrm{Co}$ source by comparing the relative positions of the $6.4 \mathrm{keV} \mathrm{Fe}$ $\mathrm{K}_{\alpha}$ line and the $14.4 \mathrm{keV} \gamma$ line. A comparison of three such spectra measured at different temperatures and different gains is presented in Fig. 12.

The amplitude ratio of the 14.4 and $6.4 \mathrm{keV} \mathrm{X}-$ ray signals versus gain, measured at -20 and $1{ }^{\circ} \mathrm{C}$, 


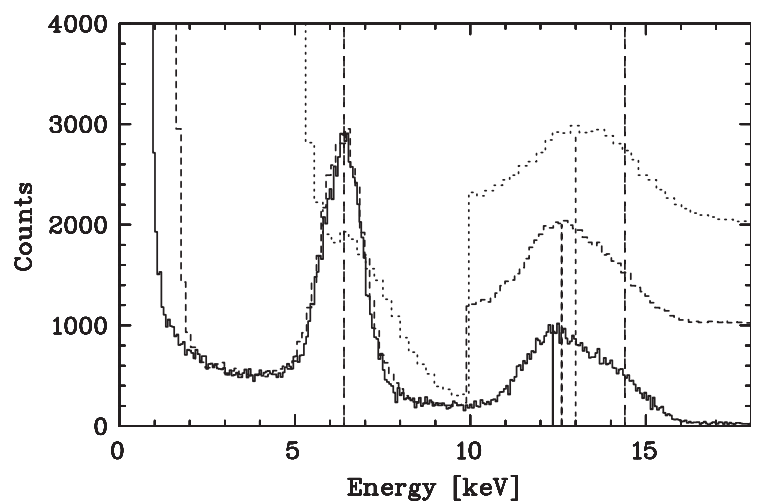

Fig. 12. LAAPD energy spectra from measurements of $6.4 \mathrm{keV}$ $\mathrm{X}$-rays and $14.4 \mathrm{keV} \gamma$ rays from a ${ }^{57} \mathrm{Co}$ source, performed at $-24^{\circ} \mathrm{C}$ (solid line), $1^{\circ} \mathrm{C}$ (dashed line), and $27^{\circ} \mathrm{C}$ (dotted line) at gains of 400, 350, and 200, respectively. Each spectrum was separately calibrated in energy such that the $6.4 \mathrm{keV}$ peak occurs at the same position. The maxima of the $14.4 \mathrm{keV}$ peaks are then at $12.4,12.6$, and $13.0 \mathrm{keV}$, respectively, due to the nonlinear LAAPD response. The parts of the spectra above $10 \mathrm{keV}$ for the measurements at 1 and $27^{\circ} \mathrm{C}$ are shifted in the $y$ direction by 1000 and 2000, respectively.

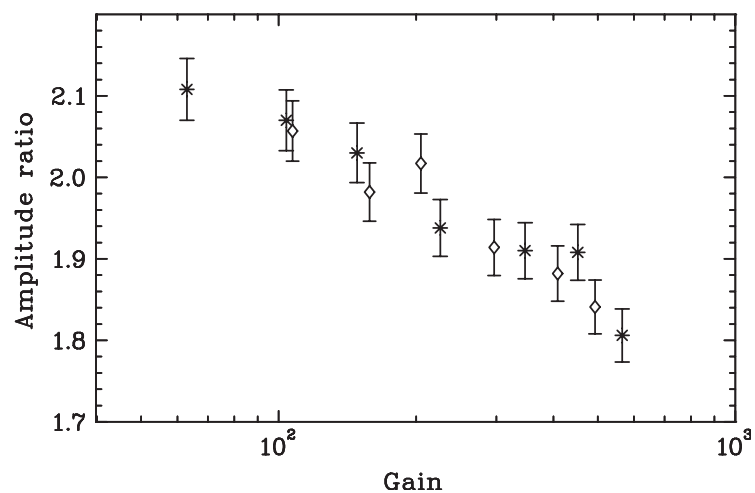

Fig. 13. Ratio of the amplitudes of 14.4 and $6.4 \mathrm{keV}$ signals versus LAAPD gain, measured at $-20(\diamond)$ and $1{ }^{\circ} \mathrm{C}(*)$.

is shown in Fig. 13. The LAAPD nonlinear response for X-rays with different energies is temperature independent; normalized to the value for linear operation, $14.4 / 6.4$, the nonlinear effect is $11 \%$ at gain 200 and $16 \%$ at gain 400 .

The ratio of the X-ray to visible-light gains, shown in Fig. 14, was measured by a simultaneous

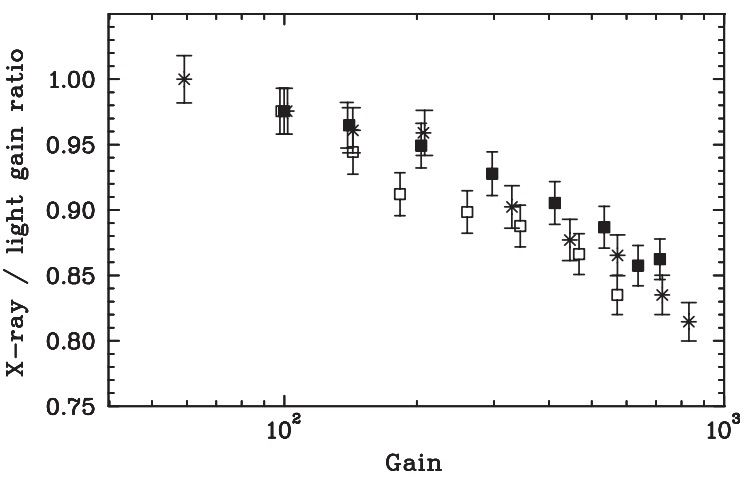

Fig. 14. Ratio of $5.4 \mathrm{keV} \mathrm{X}$-ray to visible-light gains versus LAAPD gain, measured at $-12(\square), 1(*)$, and $10^{\circ} \mathrm{C}$

illumination of the LAAPD by $5.4 \mathrm{keV}$ X-rays and by visible-light pulses. A possible temperature dependence of this ratio, as was observed for the API LAAPDs [6], is not visible given our measurement uncertainty. The nonlinearity effect is $5 \%$ at a gain of 200 , and reaches $10 \%$ at a gain of 400. Assuming that the LAAPD response to the light pulses with an energy equivalent of $11-14.5 \mathrm{keV}$ is linear, one can consider the nonlinearity as an absolute nonlinearity for $5.4 \mathrm{keV}$ X-rays.

Assuming that the nonlinearities for 5.4 and $6.4 \mathrm{keV} \mathrm{X}$-rays are similar, the absolute nonlinearity for $14.4 \mathrm{keV} \mathrm{X-rays} \mathrm{can} \mathrm{be} \mathrm{estimated} \mathrm{from} \mathrm{the}$ measurements shown in Figs. 13 and 14. It amounts to about $15 \%$ at a gain of 200 and to about $24 \%$ at a gain of 400 .

The high local charge density created in the LAAPD by an interacting X-ray-the reason for the nonlinear LAAPD response-is proportional to the number of electron-hole pairs, and hence, at a given LAAPD gain, to the X-ray energy. It is thus reasonable to assume that the nonlinearity at a certain gain is, in first order, proportional to the $\mathrm{X}$-ray energy. The ratio of the nonlinearities for $14.4 \mathrm{keV}$ X-rays to $5.4(6.4) \mathrm{keV}$ $\mathrm{X}$-rays is 3.0 and 2.4 for gains 200 and 400, respectively. These ratios differ from the direct ratio of the energies $14.4 / 5.4(6.4)=2.7(2.3)$ by less than $15 \%$, an error introduced by the assumption of the same nonlinearity for 5.4 and $6.4 \mathrm{keV}$ X-rays. 


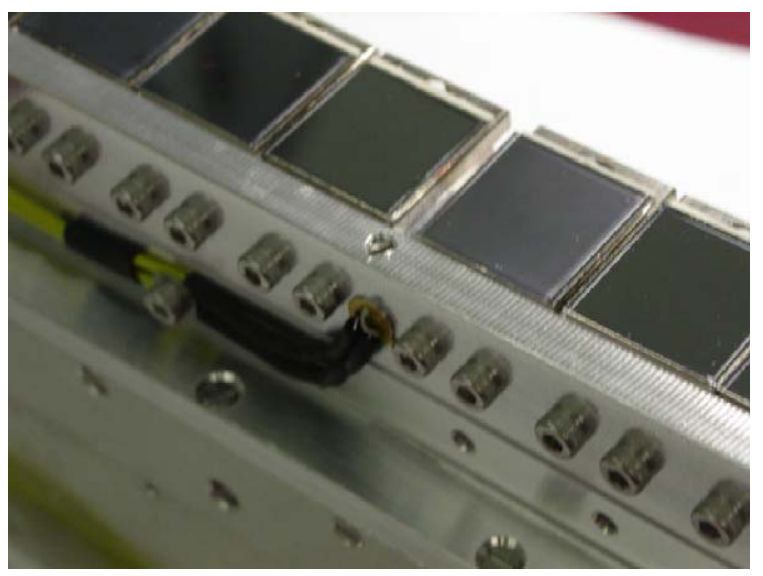

Fig. 15. Central part of one RMD LAAPD array. The wires are the thermometer leads electrical connections. High-voltage connections and preamplifiers are located on the backside of each LAAPD.

\section{LAAPD application in the muonic $2 S$ Lamb shift experiment}

\subsection{LAAPD operation conditions}

During the most recent data-taking period of the muonic 2S Lamb shift experiment in 2003, two face-to-face rows of ten RMD LAAPDs each were mounted around the target. Fig. 15 shows the central part of one row. The detector assembly was operated in a vacuum of $10^{-6}$ mbar and a magnetic field of $5 \mathrm{~T}$.

For an optimal measurement of the $1.9 \mathrm{keV} \mathrm{X-}$ ray line, we cooled the whole mount to $-30^{\circ} \mathrm{C}$ by circulating cold methanol through a small heat exchanger which was in thermal contact with the LAAPDs. The resolutions (FWHM) obtained for $5.9 \mathrm{keV}$ X-rays varied for the 20 LAAPDs between $11 \%$ and $20 \%$, with an average of about $15 \%$. A tendency that LAAPDs with higher gain at a given high voltage have better resolution was observed.

The typical bias voltage was around $1600 \mathrm{~V}$ and the corresponding gain about 400 , a value chosen for each LAAPD so that the amplitude of the $1.9 \mathrm{keV}$ X-ray signal was sufficiently above the noise level without worsening the resolution. Based on the discussion in Section 2.7 the nonlinearity for X-rays with energies $\sim 2 \mathrm{keV}$ can be estimated to be about $3-4 \%$ at gain 400 . The

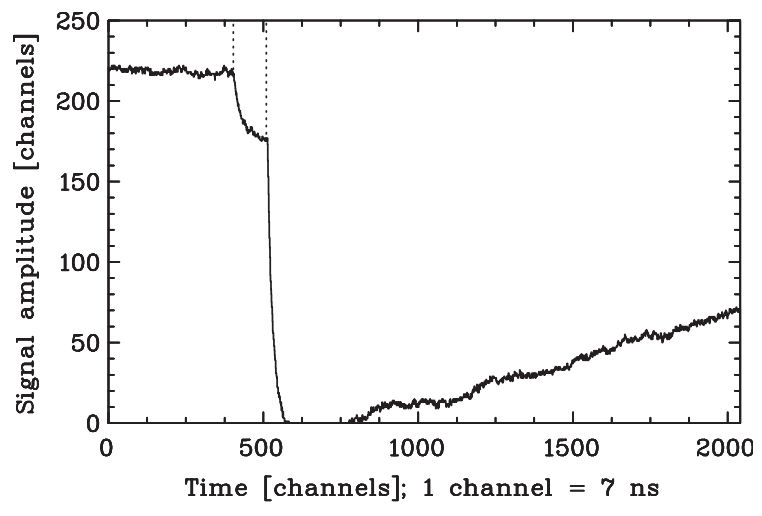

Fig. 16. A typical event with two superimposed LAAPD pulses $(\Delta t=750 \mathrm{~ns})$ recorded by a wave-form digitizer. The pulses have negative amplitude. The small first pulse is a $1.9 \mathrm{keV} \mathrm{X}$-ray signal and the second larger pulse is due to a muon-decay electron. The digitizer dynamic range is between 0 and 255, and hence the second pulse is saturated between channels 600 and 800 and only gives 0 as reading. The beginning of each signal is marked by a vertical dotted line.

dark current was between 8 and $25 \mathrm{nA}$ for the majority of the LAAPDs.

After the preamplifiers, the amplitude of a $1.9 \mathrm{keV} \mathrm{X}$-ray signal was about $2 \mathrm{mV}$. The signal rise time for $25 \%$ of the detectors was below $\sim 250 \mathrm{~ns}$, for $50 \%$ was in the interval $300-450 \mathrm{~ns}$ and for $25 \%$ was above $450 \mathrm{~ns}$. After further amplification, the negative amplitude signals were stored in an 8-bit wave-form digitizer [14] operated at $140 \mathrm{MHz}$, which allowed an optimal suppression of background signals with nonstandard shape and, in particular, permitted the separation of two consecutive, superimposed pulses. A typical event from one LAAPD is given in Fig. 16. The baseline noise fluctuations, although small, cannot be neglected in comparison with the amplitude of the $2 \mathrm{keV} \mathrm{X}$-ray.

\section{2. $L A A P D$ response to $\alpha$ particles}

In our experiment, the LAAPDs were exposed to $\alpha$ particles with kinetic energies from 2 to $9 \mathrm{MeV}$ at a rate of about $5 \mathrm{~s}^{-1}$. The $\alpha$ particles came from the dielectric coating of two high-reflectivity laser mirrors for $6 \mu \mathrm{m}$ light which contain thorium. The mirrors were mounted only $1.5 \mathrm{~cm}$ away from the LAAPD surface. 


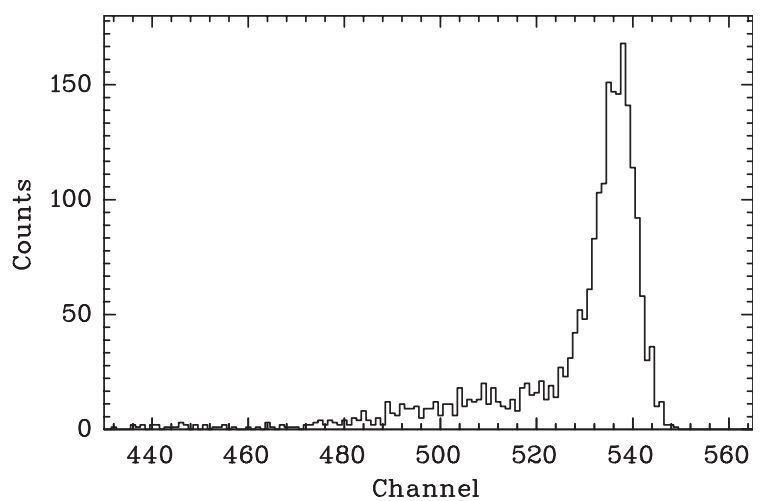

Fig. 17. LAAPD energy spectrum of the $\alpha$ particles from a ${ }^{241} \mathrm{Am}$ source measured at $600 \mathrm{~V}$. Due to energy loss in the source, the spectrum has a low energy tail.

The response of the API LAAPDs to $\alpha$ particles was studied with a collimated ${ }^{241} \mathrm{Am} \alpha$ source $(5.4 \mathrm{MeV})$ providing events at about $20 \mathrm{~s}^{-1}$. Due to the high ionization density of $\alpha$ particles, they produce signals measurable in the LAAPD even at room temperature and without bias voltage. The signal has a long rise time of about $500 \mathrm{~ns}$ and becomes faster when bias voltage is applied. At $600 \mathrm{~V}$ the rise time is about $230 \mathrm{~ns}$ and a typical $\alpha$ spectrum with a low-energy tail, due to energy losses in the source, is observed (Fig. 17).

However, with a further high voltage increase a high-energy tail appears. In addition, at about $1300 \mathrm{~V}$, huge pulses are observed which correspond to a LAAPD gain of order $10^{5}$. The origin of these pulses is attributed to a plasma discharge in the avalanche region along the very high ionization density of an $\alpha$ particle trace [15]. These pulses cause saturation of the preamplifier and have a long recovery time. In addition, the presence of these signals gives rise to large fluctuations of the LAAPD dark current. In a spectrum measured at $1300 \mathrm{~V}$ (Fig. 18), one may recognize a peak due to some $\alpha$ particles which are not creating a plasma discharge. Not visible in Fig. 18 is the saturated signal peak from the plasma discharge signals.

With an oscilloscope probe sensor we studied the shape of the plasma discharge signals after the input coupling capacitor, while the preamplifier was removed. The LAAPD was operated at

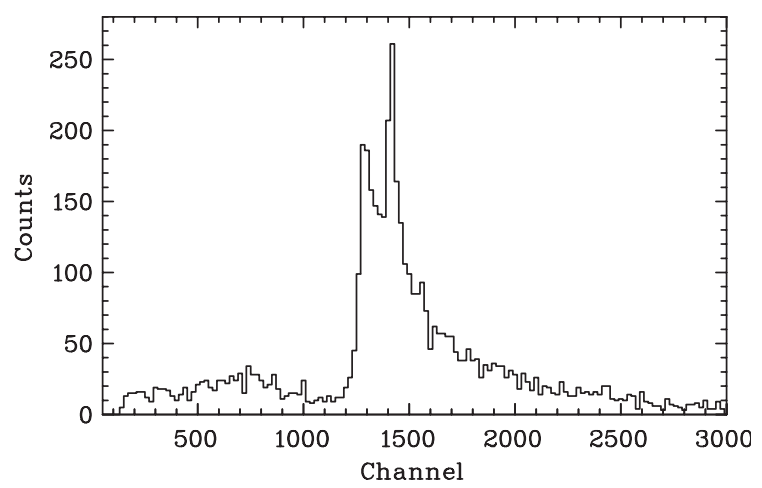

Fig. 18. LAAPD energy spectrum of the $\alpha$ particles as in Fig. 17 , but measured at $1300 \mathrm{~V}$. It corresponds to not saturated signals. Note the high-energy tail which is absent in Fig. 17.

$1780 \mathrm{~V}$. The saturated signal has a long relaxation time of $200 \mathrm{~ms}$ and a huge amplitude of $70 \mathrm{~V}$. For comparison, a $6 \mathrm{keV} \mathrm{X}$-ray produces a signal of $\sim 10^{-4} \mathrm{~V}$ in amplitude. Such a long recovery time represented an unacceptable dead time and the high amplitude was dangerous for the preamplifier.

Two high-conductance ultra fast diodes $1 \mathrm{~N} 4150$ [16], chosen for their fast recovery time of around $5 \mathrm{~ns}$, were installed at the input of the charge integrating RAL 108A preamplifier [10] to limit the amplitude of plasma discharge signals. To reduce the relaxation time, another input coupling capacitor was added and placed after the two diodes. Both input coupling capacitances were tuned in order to find an optimum between shortening the relaxation time and losing the charge sent to the preamplifier. A good compromise was found by using a first capacitance of $4.7 \mathrm{nF}$ and a second of $2.2 \mathrm{nF}$. The electronic scheme in Fig. 19 represents the final arrangement of the preamplifier electronics. The recovery time was reduced by a factor of 10 to reach $20 \mathrm{~ms}$, measured after the preamplifier.

At lower temperatures, the characteristics of plasma discharge signals did not change. The only difference was that they started to appear at lower voltages, since at lower temperatures a certain gain is reached already at a lower voltage.

In our application during the 2002 data-taking period, after having installed the laser mirrors and, hence, the $\alpha$ particles source, three out of the ten 


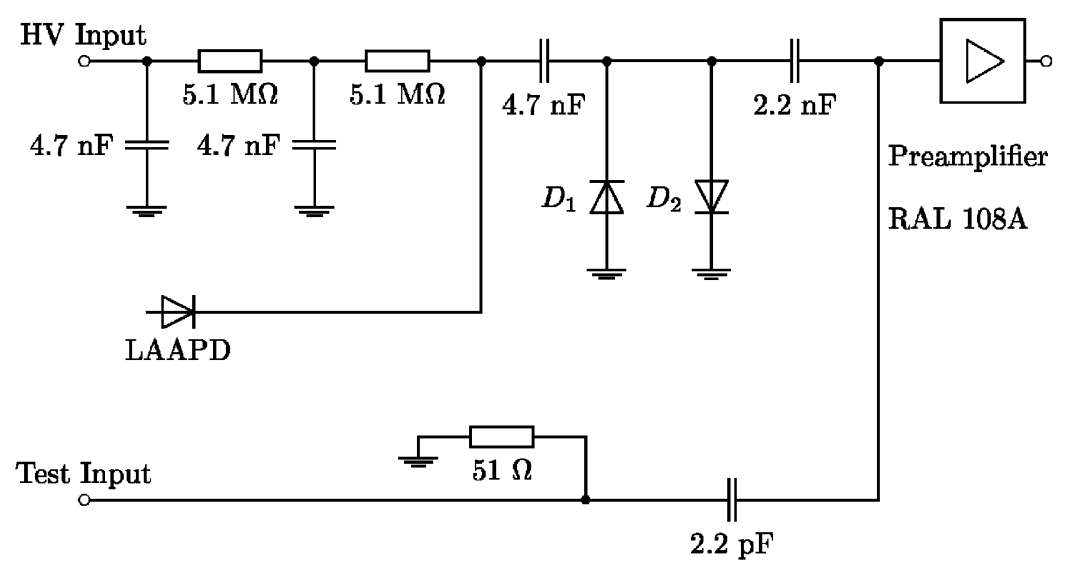

Fig. 19. Final electronic scheme of a preamplifier box used for each RMD LAAPD. Note the two input coupling capacitors (4.7 $\mathrm{nF}$ and $2.2 \mathrm{nF})$ with the two diodes $\left(\mathrm{D}_{1}, \mathrm{D}_{2}\right)$ in between; for an explanation see text.

API LAAPDs were destroyed within a day. This happened after two weeks of perfect functioning, so there was a high probability that the API LAAPDs were destroyed by the $\alpha$ particles.

For the 2003 data-taking period we used RMD LAAPDs as X-ray detectors. Due to their rectangular shape and to only a thin border of inactive material, they covered a larger solid angle. With 20 LAAPDs we were able to cover an average solid angle of $30 \%$ compared to $17 \%$ obtained with the API LAAPDs. Unfortunately, when exposed to $\alpha$ particles, their response was very similar to that of the API LAAPDs. To avoid the risk of LAAPD damage or slow deterioration, during the final data taking we shielded the RMD LAAPDs with a $0.2 \mathrm{~mm}$ thick lithium sheet, absorbing all $\alpha$ particles below $9 \mathrm{MeV}$, but unavoidably also about $40 \%$ of the $1.9 \mathrm{keV}$ X-rays.

\section{Discussion and conclusions}

The experiment measuring the $2 \mathrm{~S}$ Lamb shift in muonic hydrogen, performed at the Paul Scherrer Institute in Switzerland, has demanding requirements for the $1.9 \mathrm{keV} \mathrm{X}$-ray detectors. The beveled-edge API LAAPDs used in the initial stages of the experiment were replaced by the planar RMD LAAPDs for the latest data taking.

In our experiment the LAAPDs are exposed to $\alpha$ particles causing high-amplitude signals with a long recovery time and, with a high probability, detector damage or destruction. No significant difference in the detector response to $\alpha$ particles was found in between API and RMD LAAPDs.

The results of systematic tests studying the RMD LAAPDs performance at low temperatures were shown. In comparison, the API LAAPDs [6] show, in general, better performance and require less or no cooling, but the much higher solid angle coverage achievable with the RMD LAAPDs is an essential advantage for an application like ours.

In order to perform any reasonable soft X-ray spectroscopy measurements, the RMD LAAPDs have to be cooled. The dark current of RMD LAAPDs is of the order of a few $\mu \mathrm{A}$ at room temperature whereas values around $10 \mathrm{nA}$ are reached at $-30^{\circ} \mathrm{C}$. In contrast, the dark current of the API LAAPDs is of the order of a few hundred $\mathrm{nA}$ at room temperature and can be reduced to $10 \mathrm{nA}$ already at $0^{\circ} \mathrm{C}$.

The RMD LAAPDs we used demonstrated worse energy resolution in comparison with the API LAAPDs. An 8\% energy resolution for visible light with an energy equivalent of about $10 \mathrm{keV}$ was obtained at room temperature with the API LAAPDs; to reach the same resolution with the RMD LAAPDs, they needed to be cooled to $-10^{\circ} \mathrm{C}$. With the API LAAPDs an energy resolution of $11 \%$ for $5.4 \mathrm{keV} \mathrm{X}$-rays at room temperature was measured; with the RMD LAAPDs the 
resolution of $11 \%$ for $5.9 \mathrm{keV} \mathrm{X}$-rays was reached only at $-30^{\circ} \mathrm{C}$ with the best LAAPDs.

The API LAAPDs operate with their optimal resolution at gain 50 . At this gain the nonlinearity for $5.4 \mathrm{keV} \mathrm{X-rays} \mathrm{is} \mathrm{negligible} \mathrm{and} \mathrm{at} \mathrm{gain} 200$ it reaches only $1 \%$. RMD LAAPDs have their best resolution at gain 200. At this gain the nonlinearity for $5.4 \mathrm{keV} \mathrm{X}$-rays is about $5 \%$, and at gain 400 reaches $10 \%$. The relatively high nonlinearity of the RMD LAAPDs is not an essential problem for our experiment, because we are interested only in the intensity variation of the $1.9 \mathrm{keV} \mathrm{X}$-rays as a function of the laser frequency. A higher gain achievable with the RMD LAAPDs in feasible Xray spectroscopy measurements represents an advantage, especially for an experiment realized in a high-noise environment.

Our final data taking was performed at typical gains of 400 with a satisfactory energy resolution. All 20 RMD LAAPDs were operated for several weeks without any deterioration. At the end it was proved that the RMD LAAPDs are suitable for soft X-ray spectroscopy measurements.

\section{Acknowledgements}

Support is acknowledged from the Swiss National Science Foundation, the Swiss Academy of Engineering Sciences, from the Portuguese Foundation for Science and Technology (FCT) Lisbon, and FEDER through the Project POCTI/FNU/ 41720/2001, and the program PAI Germaine de Staël no. 07819NH du Ministère des Affaires Étrangères France. The project was in part supported by the US Department of Energy.
Laboratoire Kastler Brossel is a Unité Mixte de Recherche du CNRS no. 8552. Financial support for this research was received from the Swedish Science Research Councils (VR).

The authors would like to thank L. Simons and B. Leoni for setting up the cyclotron trap. We also thank the PSI accelerator division, PSI Hallendienst, PSI and University Fribourg workshops, and other support groups for their valuable help.

\section{References}

[1] F. Kottmann, et al., Hyp. Interact. 138 (2001) 55.

[2] Advanced Photonix Inc., 1240 Avenida Acaso, Camarillo, CA 93012, USA.

[3] M. Moszynski, M. Szawlowsky, M. Kapusta, M. Balcerzyk, Nucl. Instr. and Meth. A 485 (2002) 504.

[4] L.M.P. Fernandes, et al., Nucl. Instr. and Meth. A 498 (2003) 362.

[5] M. Boucher, et al., Nucl. Instr. and Meth. A 505 (2003) 136.

[6] L.M.P. Fernandes, et al., IEEE Trans. Nucl. Sci. NS- 51 (4) (2004) 1575.

[7] L.M.P. Fernandes, et al., Spectro. Acta B 58 (2003) 2255.

[8] Radiation Monitoring Devices, Inc., 44 Hunt Street, Watertown, MA 02472, USA.

[9] R. Farrell, et al., Nucl. Instr. and Meth. A 442 (2000) 171.

[10] CLRC Rutherford Appleton Laboratory, Chilton, Didcot, Oxfordshire, OX11 0QX, UK.

[11] M. Moszynski, M. Kapusta, M. Szawlowski, D. Wolski, Nucl. Instr. and Meth. A 442 (2000) 230.

[12] J.P. Pansart, Nucl. Instr. and Meth. A 387 (1997) 186.

[13] M. Moszynski, M. Szawlowski, M. Kapusta, M. Balcerzyk, Nucl. Instr. and Meth. A 497 (2003) 226.

[14] S. Dhawan, Developed at Physics Department, Yale University, New Haven, CT 06520-8121, USA.

[15] R. Farrell, Radiation Monitoring Devices, Private communication.

[16] Fairchild Semiconductor Corporation, 82 Running Hill Road, South Portland, ME 04106, USA. 